Gut, 1981, 22, 228-233

Clinical trial

\title{
Randomised trial of endoscopic argon laser photocoagulation in bleeding peptic ulcers
}

\author{
A G VALLON, P B COTTON,* B H LAURENCE, $\dagger$ J R ARMENGOL MIRO, AND \\ J C SALORD OSES
}

From the Gastrointestinal Unit, The Middlesex Hospital, London, and

Ciudad Sanitaria de la Seguridad Social, Francisco Franco, Barcelona, Spain

SUMMARY Emergency endoscopy on 332 patients with acute upper gastrointestinal bleeding showed that 178 had peptic ulcers; 28 of these were actively bleeding (spurting) and 108 showed stigmata of recent haemorrhage (vessels or spots in the ulcer base) suggesting a risk of rebleeding. These 136 patients were randomly allocated to Argon laser photocoagulation or to no additional therapy (controls) at the time of initial endoscopy. All patients received conventional management, and the controlling clinicians did not know whether or not the laser had been used in any individual patient. The laser system proved both simple and safe in use. Initial haemostasis was achieved by the laser in 10 of 15 'spurting vessels', but four of 13 'control' spurting vessels also stopped bleeding spontaneously. Overall, there were no statistically significant differences between the laser treated and control groups in terms of rebleeding, the need for surgical intervention, or death. These results require amplification in larger trials, and comparison with other studies using different protocols and other haemostatic methods.

Emergency fibreoptic endoscopy is now widely used in patients with acute upper gastrointestinal bleeding $^{1}$ and can provide accurate diagnostic ${ }^{2}$ and prognostic information, ${ }^{3}$ facilitating decisions about the need for surgical intervention. Despite this, there is no real evidence that overall results have improved ${ }^{4}$ and patients continue to die. Some bleed as the terminal event of an incurable disease; in others, the nature of the bleeding lesion, such as gastric cancer or oesophageal varices, indicates a poor prognosis even when bleeding can be controlled. Most patients with superficial lesions stop bleeding spontaneously. It is bleeding peptic ulcers which pose the main clinical challenge. The outlook is excellent if haemostasis can be achieved; yet many such patients are elderly, and withstand emergency surgery poorly. 5

Many alternatives to surgical intervention have

-Address for reprint requests: Dr P B Cotton, Gastrointestina Unit. The Middlesex Hospital, London W1N 8AA.

+Present address: Sir Charles Gairdner Hospital, Perth, Western Australia.

Received for publication 22 September 1980 been proposed, and there is increasing interest in endoscopic methods of haemostasis ${ }^{6}$ - of which direct coagulation using electrodiathermy ${ }^{7}$ or a laser beam $^{8}$ appears to be the most promising. Many workers in this field have been concerned about the possible risks of electrodiathermy; the depth of injury is difficult to control, and the resulting coagulum is easily dislodged. By contrast, a laser beam allows coagulation without touching the lesion, and extensive animal experimentation shows that haemostasis can be achieved without full thickness damage to the gastric wall. ${ }^{9-11}$ Preliminary reports suggest that endoscopic laser photocoagulation is also safe and effective in man. ${ }^{812}$ We recently collected experience of argon laser photocoagulation in several European centres; the technique stopped active bleeding from peptic ulcers in $\mathbf{4 8}$ to 60 patients with a low incidence of rebleeding. ${ }^{13}$ However, many ulcers stop bleeding spontaneously; the real clinical contribution of the method can be established only by a randomised trial.

Large numbers of patients are required for such a study because mortality rates are already relatively low, and because many different types of lesion are 
involved. Few hospitals admit more than 200 bleeding patients annually; multicentre trials are in progress, but these carry their own problems. We have undertaken a randomised study at the Francisco Franco Hospital in Barcelona, Spain, where more than 1000 emergency endoscopies are performed each year for acute upper gastrointestinal bleeding.

\section{Methods}

Ethical approval for this project was given by the Clinical Investigations Panel of The Middlesex Hospital and Medical School, and by the Director of the Department of Surgery, Ciudad Sanitaria de la Seguridad Social, Francisco Franco, Spain.

\section{EMERGENCY ENDOSCOPY}

The endoscopy unit of the Francisco Franco Hospital is situated alongside the emergency department. All severely bleeding patients were referred immediately after resuscitation; some patients with less severe bleeding were referred after transfer to the wards, but this study includes only those patients examined within 24 hours of admission or the last episode of bleeding. Gastric lavage was not used routinely. Endoscopy was performed, usually without sedation, by one of three experienced endoscopists using Olympus fibrescopes-an experimental doublechannelled forward viewing instrument, or the fore-oblique model GIFK. All bleeding lesions and those with attached clot were washed thoroughly with a jet of water applied via the endoscope channel.

\section{TRIAL INCLUSIONS AND RANDOMISATION}

The trial concerned only those patients with endoscopically proven acute or chronic peptic ulcers. Because of their different potentials for re-bleeding ${ }^{3}$ ulcers were divided into four groups, according to the appearances at emergency endoscopy: (1) active bleeding (spurting); (2) protruding vessels; or (3) visible spots in the ulcer base after washing; and (4) those with no stigmata of recent haemorrhage (clean bases). The latter group of patients have a negligible risk of rebleeding, ${ }^{3}$ and were excluded from the trial. Randomisation into laser treatment or the control group was performed for the remainder of the patients at the time of endoscopy, after identifying a suitable ulcer, using numbered cards; patients with gastric and duodenal ulcers were stratified separately; anastomotic ulcers were classified as duodenal.

\section{LASER TREATMENT}

We used the Spectra-Physics model 770 argon ion laser with a maximum power output of $12 \mathrm{~W}$. The beam was transmitted through a flexible quartz fibre $(400 \mu$ diameter) lying within a $2 \mathrm{~mm}$ Teflon catheter, which also allowed passage of a jet of carbon dioxide to disperse blood, and to keep the fibre clean. Photocoagulation was carried out with a power output of 6-10 W (using the highest power for those ulcers with the most severe bleeding) in one to two second bursts for a total of 15-100 seconds. After photocoagulation the ulcers were irrigated with water and observed endoscopically for five minutes to detect rebleeding.

\section{AFTER ENDOSCOPY}

All patients were admitted to the wards under the care of the 'on-call' surgeon, who remained entirely responsible for the patients' management; he was unaware whether or not laser photocoagulation had been used. All patients were given or continued on cimetidine ( $1 \mathrm{~g}$ per day) and were followed clinically for evidence of rebleeding during their stay in hospital. Endoscopic confirmation was obtained when rebleeding was in doubt. Patients in the trial received only one laser treatment.

\section{TRIAL INDICES}

The laser treatment and control groups were compared for (1) continuing bleeding and rebleeding; (2) emergency surgery for bleeding; and (3) deaths in hospital. Statistical evaluation was carried out using the Chi Square test with Yates' correction.

\section{Results}

Emergency endoscopy was performed on 332 patients while the laser was available and functioning between April and September 1979. Sources of bleeding are shown in Table 1. One hundred and thirty-six patients with ulcers which were actively bleeding, or showed stigmata of recent haemorrhage, were entered into the randomised trial. The two groups were comparable by age, severity of bleeding, and the presence of concomitant conditions. Overall results are shown in Table 2.

SPURTING ULCER S (active bleeding at time of endoscopy)

There were nine gastric and 19 duodenal 'spurting' ulcers; initial haemostasis was achieved in three of the five gastric, and in seven of the 10 duodenal ulcers allocated to laser treatment. Rebleeding occurred within 36 hours in one gastric, and one duodenal ulcer. Emergency surgery was performed in three gastric and four duodenal ulcers for bleeding, and in one duodenal ulcer because of its size. Neither death in the treatment group was attributable to further haemorrhage. In two duodenal 
Table 1 Diagnosis at emergency endoscopy

\begin{tabular}{|c|c|c|c|c|}
\hline & & & \multicolumn{2}{|c|}{ Total } \\
\hline & & & no. & $\%$ \\
\hline \multicolumn{3}{|c|}{ Ulcers (61 g, $103 \mathrm{~d}, 5$ both, 9 stomal) } & 178 & 53 \\
\hline & no. & $\%$ ulcers & & \\
\hline $\begin{array}{l}\text { Actively bleeding } \\
\text { Protruding vessels } \\
\text { Central spots }\end{array}$ & $\begin{array}{l}28 \\
35 \\
73\end{array}$ & $\begin{array}{l}16 \\
20 \\
41\end{array}$ & & \\
\hline $\begin{array}{l}\text { No stigma (clean base) } \\
\text { Exclusions" }\end{array}$ & $\begin{array}{r}39 \\
3\end{array}$ & $\begin{array}{r}21 \\
2\end{array}$ & & \\
\hline \multicolumn{3}{|l|}{$\begin{array}{l}\text { Varices } \\
\text { Mallory Weiss lesions } \\
\text { Oesophagitis } \\
\text { Gastric erosions } \\
\text { Tumours } \\
\text { Other causes } \\
\text { No diagnosis } \\
\text { Total }\end{array}$} & $\begin{array}{r}64 \\
20 \\
9 \\
22 \\
16 \\
5 \\
18 \\
332\end{array}$ & $\begin{array}{r}19 \\
6 \\
3 \\
7 \\
5 \\
1 \\
6 \\
100\end{array}$ \\
\hline
\end{tabular}

g: gastric; d: duodenal ulcer.

-Patients with multiple bleeding ulcers from the Intensive Therapy Unit.

The box indicates the patients included in the randomised trial.

ulcers, laser treatment was considered inadequate because scarring and distortion of the duodenal bulb had precluded accurate aiming.

The four spurting gastric ulcers in the control group continued to bleed; surgical haemostasis was achieved in three of them, but one patient (aged 79 years) died while being prepared for surgery.

Bleeding ceased spontaneously in four of the nine spurting duodenal ulcers, but two of these patients bled again within 24 hours; of these seven with further bleeding, six underwent emergency surgery; there was one postoperative death. One patient died without surgery in hepatic coma.

\section{ULCERS WITH PROTRUDING VESSELS}

Protruding vessels were visible in 35 ulcers (14 gastric and 21 duodenal) which were not actively bleeding at the time of endoscopy; 19 were allocated to laser treatment, and 16 to the control group. Rebleeding sufficient to require emergency surgery, or to have contributed to death, occurred in eight patients of each group. There were three deaths in each group; five were associated with rebleeding but only one of the patients had undergone surgery.

One patient developed an exacerbation of pain during laser treatment; laparotomy showed a duodenal ulcer with a covered perforation, which is believed to have occurred before endoscopy.

ULCERS WITH CENTRAL SPOTS

Rebleeding occurred rarely, but with equal frequency, in the 34.1aser treated and 39 control
Table 2 Results in laser treated $(L)$ and control $(C)$ groups according to appearance of ulcer base at time of initial endoscopy

\begin{tabular}{|c|c|c|c|c|c|c|}
\hline & \multicolumn{2}{|c|}{ Spurting } & \multicolumn{2}{|c|}{$\begin{array}{l}\text { Protruding } \\
\text { vessel } \\
\text { (not } \\
\text { spurting) }\end{array}$} & \multicolumn{2}{|c|}{ Spot } \\
\hline & $L$ & $C$ & $\boldsymbol{L}$ & $C$ & $\boldsymbol{L}$ & C \\
\hline Total & 15 & 13 & 19 & 16 & 34 & 39 \\
\hline $\begin{array}{l}\text { Initial haemostasis } \\
\text { Further }\end{array}$ & 10 & 4 & - & - & - & - \\
\hline $\begin{array}{l}\text { haemorrhage } \\
\text { Surgery for }\end{array}$ & 7 & 11 & 8 & 8 & 5 & 4 \\
\hline $\begin{array}{l}\text { bleeding } \\
\text { Surgery- }\end{array}$ & 7 & 9 & 6 & 5 & 2 & 4 \\
\hline $\begin{array}{l}\text { other indication } \\
\text { Death }\end{array}$ & $\begin{array}{l}1 \\
2\end{array}$ & $\begin{array}{l}\mathbf{0} \\
\mathbf{3}\end{array}$ & $1 *$ & $\begin{array}{l}\mathbf{0} \\
\mathbf{3}\end{array}$ & $\begin{array}{l}\mathbf{0} \\
\mathbf{0}\end{array}$ & $\begin{array}{l}1 \\
4\end{array}$ \\
\hline
\end{tabular}

Patient with covered perforation.

patients with ulcers showing only a central spot. The four deaths in the control group were not related to further bleeding, and all patients undergoing surgery survived without complication.

\section{EMERGENCY SURGERY AND DEATH}

Surgery was performed on $36(26.5 \%)$ of the 136 patients in the trial- 12 because of continued bleeding, 21 for rebleeding, and three because of the nature of the lesion (including the one perforation). Two $(5.5 \%)$ of the operated patients died.

Overall, $15(11 \%)$ of the 136 patients in the trial died; five were in the laser group, and 10 controls. While eight of the 15 patients who died had suffered further bleeding after hospital admission, only two had undergone emergency surgery. Surgery was not advised in the remaining six because of serious concomitant disease.

\section{VALUE OF STIGMATA IN PREDICTING OUTCOME}

There was one episode of rebleeding among 39 patients whose ulcers showed no stigmata of haemorrhage at the time of initial endoscopy; laparotomy in this patient revealed a bleeding chronic gastric ulcer which had been missed at endoscopy. One patient in this group died suddenly six days after admission, probably from a pulmonary embolus.

Follow-up of this group, and the controls, confirmed the prognostic significance of active bleeding and the presence of protruding vessels (Table 3). Further haemorrhage occurred with similar frequency in patients with gastric (nine of 37) and duodenal ulcers $(15$ of 70$)$.

STATISTICAL ANALYSIS OF RESULTS

There were no statistical differences between the 
Table 3 Diagnostic significance of appearance of ulcer base in untreated patients (those with no stigmata, and control patients in trial)

\begin{tabular}{lcccccc}
\hline & Total & \multicolumn{2}{c}{ Further bleeding } & \multicolumn{2}{c}{ Surgery } \\
\cline { 3 - 7 } & & $n o$ & $\%$ & & no. & $\%$ \\
\hline Actively bleeding & 13 & 11 & 85 & & 9 & 69 \\
Protruding vessel & 16 & 8 & 50 & 5 & 31 \\
Spots & 39 & 4 & 10 & 5 & 13 \\
No stigmata & 39 & $1 *$ & 2 & $1 *$ & 2 \\
Total & 107 & 24 & 22 & & 20 & 19 \\
\hline
\end{tabular}

-Lesion missed at endoscopy.

laser treated and control groups when considering the incidence of rebleeding, surgery, or death, whatever the type of ulcer or its appearance at the time of endoscopy.

There was a trend towards benefit from laser photocoagulation in the patients with actively bleeding (spurting) ulcers (Table 2); indeed the results for immediate and long-term haemostasis in these patients do reach conventional levels of statistical significance $(\mathrm{P}<0.05)$ when we exclude the two patients in whom laser treatment was technically difficult because of inadequate access in a deformed duodenal bulb.

\section{Discussion}

Acute upper gastrointestinal bleeding remains a common medical emergency. Despite this, current management is largely anecdotal; very few treatment trials have been performed, and this study highlights some of the problems involved. Bleeding occurs from many different lesions, and patients of different ages and states of health vary in their toleration of further bleeding and surgery. Emergency endoscopy has made it easier to identify the precise causes of bleeding, ${ }^{2}$ and therefore to study more satisfactorily the behaviour of defined groups of patients. We have concentrated on bleeding from peptic ulcers because they are the commonest cause, and effective haemostasis should permit a return to normal health. We have confirmed and extended previous suggestions ${ }^{314} 15$ that the precise endoscopic appearance of an ulcer is an important guide to prognosis. Contrary to some previous opinions, ${ }^{1}$ gastric and duodenal ulcers showed a similar tendency to continue bleeding or to bleed again, but the most striking fact was that rebleeding was far more common in ulcers with active bleeding or visible vessels than in those whose bases were clean or showed only a small central spot. This type of information indicates which groups of patients are worth entering into a treatment trial, and how they should be stratified. However, the relative sizes of these different 'stigmata' groups and their precise prognostic significance also depends upon the timing of the endoscopy. Departments with active investigation policies see more 'spurting' ulcers than those which delay; indeed, all patients would show active bleeding if endoscopy could be performed at the moment when bleeding begins. Only $16 \%$ of the ulcers in this series were spurting at the time of endoscopy, and these patients contributed only one-third of the total deaths. Herein lies the justification for attempting prophylactic treatment of non-bleeding ulcers, in the hope of reducing the risk of rebleeding.

We chose to use the argon-ion laser because of its demonstrated safety and efficiency in acute animal experiments, ${ }^{913}$ and because of the high success rate for stopping active bleeding (48/60 patients) which was reported recently from various European centres. ${ }^{13}$

This study confirms that the Spectra-Physics argon-ion laser is simple to use, safe, and can stop active bleeding. Immediate haemostasis was achieved in 10 of 13 patients with spurting ulcers, when access was adequate. However, we also showed that active bleeding can stop spontaneously (as in four of our 13 control patients), which re-emphasises the importance of randomised studies. There was a trend towards haemostatic benefit for the laser-treated patients with actively bleeding ulcers, and the difference does just reach statistical significance if we exclude the two patients with such duodenal deformity that coagulation could not be applied effectively. Apart from this, there were no statistically significant differences between the laser and control groups in any prognostic category, with regard to immediate or long-term haemostasis, the need for surgical intervention, or death in hospital.

This study was relatively small, and it is possible that larger numbers would demonstrate a benefit for laser treatment in patients with actively bleeding ulcers. However, our results indicate clearly that prophylactic treatment of non-bleeding lesions is not worth while.

We consider that the argon ion method was used optimally in this study, and that the poor results cannot be attributed to inferior technique. However, it is possible that different results would be obtained using the same equipment and a different protocol, or with an alternative form of laser. Our protocol allowed only a single attempt at laser photocoagulation. It would be as logical to treat those patients with active bleeding at initial endoscopy, and to repeat treatments in all those who bled again thereafter. Such a protocol might have provided 
different results in our own study, as eight of the 15 patients died from further bleeding, and only two of them have been judged suitable for surgical intervention. It is particularly important to have a control group in any protocol which includes multiple laser treatments, as this would increase the potential hazards, which have not been adequately emphasised. Any prolonged endoscopy must carry some hazard in seriously ill patients, including the risk of pulmonary aspiration of gastric content. Prolonged and unsuccessful attempts to obtain haemostasis may be a disadvantage to the patient simply by delaying more effective methods such as surgical intervention; surgery may prove more difficult eventually in a patient with intestines distended with gas.

It could be that the argon-ion laser is not sufficiently powerful. The Neodymium Yag laser ${ }^{1011}$ has greater penetration, which should facilitate haemostasis in larger vessels. Uncontrolled clinical results are encouraging $;{ }^{12}$ however, the use of this laser does carry a small risk of perforation, which increases the need for a randomised evaluation of its value.

The final and crucial point concerns cost-effectiveness. Even if shown to have definite haemostatic value, laser treatment would be applicable only to a small percentage of patients admitted to hospital with bleeding, perhaps not more than one patient each month in the average general hospital. Most departments would find the capital outlay (at least $£ 20000$ ) and maintenance costs difficult to justify. This economic fact would encourage aggregation of bleeding patients into a small number of hospitals, and it is entirely possible that the development of 'bleeding units' might alone do more to improve the results, by better application of known methods, than any single form of treatment. ${ }^{16}$ Such cost considerations also emphasise the need for further evaluation of simpler haemostatic techniques. Electrodiathermy probes are available in most endoscopic units. Despite some concern about the safety of diathermy in patients with acute bleeding, preliminary published results are encouraging. ${ }^{77}$ A modification which allows electrocoagulation without touching the lesion is particularly interesting ${ }^{18}$ and other ingenious techniques have been described. ${ }^{19} 20$

This study has further defined the factors infiuencing the natural history of bleeding from different lesions, and illustrates some of the difficulties in designing trial protocols. Our disappointing results need amplification, and comparison with other studies using different protocols, and other coagulation methods; emergency surgery also requires careful controlled evaluation. ${ }^{21}$ These trials will be difficult to perform and to evaluate.
We wish to acknowledge the invaluable co-operation of those surgeons who participated in the trial, especially Dr Diez Gascon, Director of Surgery, Francisco Franco Hospital, Barcelona. The work was generously supported by the Spectra-Physics company, California; Smith Kline and French, Spain; and The Wellcome Trust, London.

\section{References}

${ }^{1}$ Thomas GE, Cotton PB, Clark CG, Boulos PB. Survey of management of acute upper gastrointestinal haemorrhage. J Roy Soc Med 1980; 73: 90-5.

${ }^{2}$ Cotton PB. Gastrointestinal bleeding; endoscopy. In: Truelove SC, Goodman MJ, eds. Topics in gastroenterology. Oxford: Blackwell, 1975.

${ }^{3}$ Foster DN, Miloszewski KJA, Losowsky MS. Stigmata of recent haemorrhage in diagnosis and prognosis of upper gastrointestinal bleeding. $\mathrm{Br} \operatorname{Med} J$ 1978; 1: 1173-7.

${ }^{4}$ Dronfield MW, McIllmurray MB, Ferguson R, Atkinson $\mathbf{M}$, Langman MJS. A prospective randomised study of endoscopy and radiology in acute upper gastrointestinal tract bleeding. Lancet 1977: 1: 1167-9.

${ }^{5}$ Allan R, Dykes P. A study of the factors influencing mortality rates from gastrointestinal haemorrhage. Q J Med 1976; 45: 533-50.

${ }^{6}$ Katon RM. Experimental control of gastrointestinal haemorrhage via the endoscope: a new era dawns. Gastroenterology 1976; 70: 272-7.

${ }^{7}$ Papp JP. Endoscopic electrocoagulation of actively bleeding arterial upper gastrointestinal lesions. Am J Gastroenterol 1979; 71 : 516-21.

${ }^{8}$ Fruhmorgen P, Bodem F, Reidenbach HD, Demling L. Was ist gesichert in der laserkoagulation zur stillung gastrointestinaler blutungen? Internist 1978; 19: 707-12.

${ }^{9}$ Bown SG, Salmon PR, Kelly DS, et al. Argon laser photocoagulation in the dogs stomach. Gut 1979; 20: 680-7.

${ }^{10}$ Silverstein FE, Protell RL, Gilbert DA, et al. Argon vs Neodymium YAG laser photocoagulation of experimental canine gastric ulcers. Gastroenterology 1979; 77: 491-6.

${ }^{11}$ Dixon JA, Bevenson MM, McCloskey DW. Neodymium-Yag laser treatment of experimental canine gastric bleeding. Gastroenterology 1979; 77: 647-51.

${ }^{12}$ Kiefhaber P, Mortiz K, Schildberg FW, Feifal G, Herfarth C. Endoscopic Neodymium-Yag laser irradiation for control of bleeding acute and chronic ulcers. Langenbecks Arch Surg 1978; 347: 567-71.

${ }^{13}$ Laurence BH, Vallon AG, Cotton PB et al. Endoscopic laser photocoagulation for bleeding peptic ulcers. Lancet 1980; 1 : 124-5.

${ }^{14}$ Griffiths WJ, Neumann DA, Welsh JD. The visible vessel as an indicator of uncontrolled or recurrent gastrointestinal haemorrhage. $N$ Engl J Med 1979; 300: 1411-3.

${ }^{15}$ Nudel J, Guarena J, Milman PJ et al. Endoscopic diagnosis of active bleeding; a prognostic sign in upper 
gastrointestinal haemorrhage (abs). Gastrointest Endosc 1977; 23 : 237.

${ }^{16}$ Hunt PS, Hansky J, Korman MG. Mortality in patients with haematemesis and melaena. $B r \operatorname{Med} J$ 1979; 1: 1238-40.

${ }^{17}$ Gaisford WD. Endoscopic electro-haemostasis of active upper gastrointestinal bleeding. Am J Surg 1979; 137: 47-53.

${ }^{18}$ Matek W, Fruhmorgen P, Kaduk B, Reidenbach HD, Bodem F, Demling L. Modified electrocoagulation and its possibilities in the control of gastrointestinal bleeding. Endoscopy $1979 ; 4: 253-8$.

${ }^{19}$ Linscheer WG, Fazio TL. Control of upper gastrointestinal haemorrhage by endoscopic spraying of clotting factors. Gastroenterology 1979; 77 : 642-6.

${ }^{20}$ Silverstein F. A staunch approach to endoscopic therapy. Gastroenterology (editorial) 1979; 77: 797-8.

${ }^{21}$ Dronfield MW, Atkinson M, Langman MJS. Effect of different operation policies on mortality from bleeding peptic ulcer. Lancet 1979; 1 : 1126-8. 